\title{
Serum Response Factor in muscle tissues: from development to ageing
}

\author{
Dario Coletti (1,2), Nissrine Daou (1), Medhi Hassani (1), Zhenlin Li (1), Ara Parlakian (1)
}

(1) Sorbonne University, UPMC, Department of Biological Adaptation and Ageing, IBPS, UMR 8256 CNRS, INSERM U1164, Paris, France; (2) Dept of Anatomy, Histology, Forensic Medicine \& Ortopedics, School of Medicine Sapienza University of Rome, Italy

This article is distributed under the terms of the Creative Commons Attribution Noncommercial License (CC BY-NC 4.0) which permits any noncommercial use, distribution, and reproduction in any medium, provided the original author(s) and source are credited.

\begin{abstract}
Skeletal, cardiac and smooth muscle cells share various common characteristic features. During development the embryonic mesodermal layer contribute at different proportions to the formation of these tissues. At the functional level, contractility as well as its decline during ageing, are also common features. Cytoskeletal components of these tissues are characterized by various actin isoforms that govern through their status (polymerised versus monomeric) and their interaction with the myosins the contractile properties of these muscles. Finally, at the molecular level, a set of different transcription factors with the notable exception of Serum Response Factor SRF- which is commonly enriched in the 3 types of muscle- drive and maintain the differentiation of these cells (Myf5, MyoD, Myogenin for skeletal muscle; Nkx2.5, GATA4 for cardiomyocytes). In this review, we will focus on the transcription factor SRF and its role in the homeostasis of cardiac, smooth and skeletal muscle tissues as well as its behaviour during the age related remodelling process of these tissues with a specific emphasis on animal models and human data when available.
\end{abstract}

Key Words: SRF, Cre/loxP, Muscle tissue, Muscle remodelling, Ageing

Eur J Transl Myol 2016; 26 (2): 87-92

\section{General features}

SRF, a conserved transcription factor during evolution and encoded by a single gene with the exception of zebrafish, ${ }^{1}$ is at the confluence of multiple signalling pathways controlling the transcription of immediateearly response genes and muscle-specific genes. ${ }^{2}$ It is a member of the MADS box family of transcription factors, and binds the core sequence of CArG boxes [CC (A/T) 6 GG] as a homodimer ${ }^{2}$. This binding to the core CArG box sequence seems to occur in a sequential manner as suggested by in vitro biophysical experiments. ${ }^{3}$ Despite the low intrinsic transactivating capacity, SRF is a potent regulator of gene transcription. In vivo and in silico experiments suggest the presence of over 200 SRF-regulated target genes. ${ }^{4}$ Indeed, its ability to regulate different sets of downstream target genes may depend on the promoter context and on its association with different cofactors. Among the most studied Srf cofactors are the members of the ternary complex factor family of Ets domain proteins that are activated by MAPK phosphorylation and the family of Myocardinrelated transcriptional co-factors (Myocardin, MrtfA and MrtfB). ${ }^{2,5}$ The majority of SRF target genes are involved in cell growth, migration, cytoskeletal organization and myogenesis. Extensive in vitro studies using fibroblast cells by the Treisman lab and others, allowed the identification of a robust relationship between SRF and actin dynamics. Indeed, Rho family of small GTPases that participate in the regulation of actin dynamics have been shown to control the nuclear accumulation of Srf coactivator Mrtf-A and therefore modulate Srf activity. ${ }^{6,7}$ Monomeric G-actin binds to and sequesters Srf co-factor Mrtf in the cytoplasm thereby preventing Srf activation and gene transcription. Many receptors involved in cell/ECM interactions (focal adhesions) or cell/cell interface (adherent junctions) have also been shown to affect actin dynamics and Mrtf-mediated regulation of Srf target genes. ${ }^{5}$ More recently, several studies show a major role of SRF in regulating and being regulated by various miRNA. ${ }^{8,9}$

In vivo, the importance of SRF as a crucial transcriptional regulator was evidenced by the first classical knockout model of this gene reported by Nordheim's lab. ${ }^{10}$ They showed the requirement of SRF in the formation of the mesodermal layer during the gastrulation process of embryonic development. This early lethal phenotype (E6.5-E8) precluded the 


\section{Serum Response Factor in muscle tissues}

Eur J Transl Myol 26 (2): 87-92

possibility to further study the role of SRF in organogenesis and in adult mice models. Latter, the availability of the Cre/loxP technology allowed our lab and others to generate "floxed srf" mice and inactivate the gene in an organ and time specific manner. ${ }^{11-13}$ In this review we will summarise and comment the results obtained upon the inactivation of SRF in the 3 types of muscle tissues (skeletal, cardiac and smooth muscle cells).

\section{SRF and the cardiac tissue}

\section{SRF during cardiac development}

Heart development is a complex multiple-stage process that requires the coordinated expression of transcription factors such as Nkx2.5, GATA4, SRF, MEF2, etc. At E8.5 of embryonic development the heart is a linear tube that undergoes a rightward looping at stage E9.5. At E10.5, the emergence of trabeculations in the luminal layers of ventricles enables the myocardium to increase its mass. Subsequent septation leads to the formation of a four-chambered heart. ${ }^{14}$ In this context, we were first to generate cardiac specific knockout of SRF during embryonic development using the $\beta$-MHC promoter to drive Cre expression. ${ }^{11}$ The results obtained showed the requirement of SRF for normal cardiac development and maturation as evidenced by poor trabeculations, cardiac dilatation, and a thin myocardium that were evident at stage E11.5, leading to embryonic death. ${ }^{11}$ Two other groups using different cardiac specific cre lines found similar results. ${ }^{13,15}$

\section{SRF in the adult heart}

In humans a limited number of studies investigated the behaviour of SRF during heart failure and showed the presence of dominant negative SRF protein isoforms resulting either from the upregulation of a naturally occurring SRF RNA isoform or as a result of full length SRF cleavage by Caspase-3. ${ }^{16,17}$ In both cases the alteration of full-length SRF protein levels could affect the expression of key cardiac proteins and thus contribute to the failing heart. These results suggest the importance of SRF in cardiac homeostasis in adults. Consistent with these observations, our mice model of tamoxifen inducible cardiac specific SRF knockout led to heart failure with dilated cardiomyopathy (DCM). ${ }^{18}$ This DCM was characterized as in humans by the downregulation of energetic proteins such as the muscle creatin kinase (MCK) a progressive loss in contractility and left ventricular dilation. ${ }^{18}$ Cytoskeletal architecture is also altered in these mice as evidenced by the enlargement of the intercalated disks, the rapid loss of polymerised actin and the gradual loss in the striated pattern of desmin. ${ }^{18,19}$ Moreover, Desmin, a preferential target of advanced glycation end products (AGE) in human DCM showed similar modifications in the SRF deficient mouse model. ${ }^{19}$ Taken together, these results suggest that SRF could be a key player in the remodelling process of human DCM. To our knowledge no published data on SRF expression in ageing human heart has been reported so far. Data from animal models however suggest that SRF protein expression in the heart is increased by $20 \%$ to $16 \%$ in old rats and mice as compared to young adult rats as well as its binding capacity to target sequences. ${ }^{20,21}$ This increased expression could initiate or participate in the remodelling process of the ageing cardiac tissue that is characterised by a hypertrophic phenotype. Several mice models with different levels of SRF overexpression suggest such a possibility. ${ }^{22,23}$ Indeed, constitutive overexpression of SRF gene in mouse heart leads to concentric hypertrophic cardiomyopathy. ${ }^{24}$ The requirement of SRF for such a hypertrophic response was also evidenced by the mosaic deletion of SRF in the cardiac tissue where SRF negative cardiomyocytes were unable to undergo hypertrophy and showed a thinned phenotype as compared to the neighbouring SRF positive cardiomyocytes that develop extensive hypertrophy upon mechanical stress. ${ }^{25}$ Finally mice models of high and low tamoxifen inducible SRF overexpression developed in our lab suggest that maintaining an adequate level of SRF is crucial for cardiac homeostasis. While a low level of SRF overexpression (less then 2 fold) did not have any detectable impact on cardiac function, a higher then or equal to 4 fold overexpression was enough to disturb cardiac homeostasis through the alteration of an SRF/miRNA133-a/CTGF axis and led to a fibrotic cardiac phenotype. ${ }^{22}$ Taken together the limited clinical studies and mice models of SRF point to the fact that a tight control of SRF expression levels are necessary to maintain cardiac homeostasis at different time points in life and disruption of its balanced expression could be an initiator or a major contributor in the pathological or age related cardiac remodelling process.

\section{SRF and smooth muscle cells}

\section{SRF during vascular smooth muscle development}

During embryonic development vascular smooth muscle cells (VSMC) originate mainly from lateral mesoderm derived mesenchyme with an additional contribution of neural crest cells. They are recruited around the primary capillary network to differentiate and express characteristic markers such as SM-myosin heavy chain, Sm22 and calponin. ${ }^{26}$ However, these markers are transiently expressed in cardiac and skeletal muscle during embryonic development. ${ }^{26}$ Despite extensive studies, key tissue restricted transcription factors (such as MyoD for skeletal muscle or Nkx2.5, GATA4 for cardiac muscle) implicated in VSCM differentiation have not been identified suggesting the possible absence of such tissue specific factors. Studies on DNA regulatory elements and gene promoters identified the presence of functional CArG boxes and SRF/Myocardin binding as a common feature of major VSCM specific genes. A substantial 


\section{Serum Response Factor in muscle tissues}

Eur J Transl Myol 26 (2): 87-92

specificity is thus achieved in regulating VSMC differentiation. Finally recent evidence suggests a central role for epigenetic regulation in SMC differentiation (for review see Owens 2014). ${ }^{27}$ Accordingly, inactivation of SRF in smooth muscle cells during embryonic development using $\mathrm{Sm} 22$ promoter to drive Cre expression, leads to a decrease in the number of perivascular progenitor cells as well as defects in the cytoarchitecture at E10.5 of development suggesting a crucial role for SRF in VSMC differentiation. ${ }^{13}$ The other major vascular cell type is the endothelial cell in which SRF was also invalidated. Results concerning endothelial cells are discussed elsewhere in detail. ${ }^{28,29}$

\section{SRF in adult smooth muscle cells}

Smooth muscle cells (SMCs) are present at the level of visceral organs (intestine, bladder etc.) and the vasculature. Viceral SMCs are involved in diverse process, including regulation of airway resistance, urinary bladder emptying, and gastrointestinal (GI) motility. Contractile activity of the smooth muscle layers in the gut is crucial for food propulsion and nutrient absorption. In this context, inactivation of SRF in smooth muscle using the SMC restricted tamoxifen inducible Sm22-Cre mice leads to down-regulation of many smooth muscle-specific genes in urinary bladder, and GI tract. These mice developed a severe form of visceral myopathy with a predominant GI failure phenotype presenting the features of chronic intestinal pseudo-obstruction. ${ }^{30}$ In the vascular system, smooth muscle is organised as concentric layers of cells called media and surround the layer of endothelial cells. These cells are characterised by a contractile activity that is essential to maintain a vascular tone. Smooth muscle cells respond to various extracellular signals (the autonomic nervous system and various secreted molecules) that regulate their contractile status (vasoconstriction/ vasodilation) and control blood flow and arterial pressure. The contractile and elastic properties of vascular smooth muscle cells are altered under different pathological conditions and during ageing. Indeed these cells have the capacity to reversibly modulate their phenotype from a contractile to a synthetic proliferative status. ${ }^{31}$ Two major studies using the above-mentioned Cre mice investigated the role of SRF in the VSMC either at the level of resistance arteries or at the level of large elastic arteries. The first study reported an important role of SRF in pressureinduced myogenic tone in resistance arteries. ${ }^{32}$ Resistance arteries paly an important role in the control of local blood flow in peripheral tissues. In the absence of SRF resistance arteries show a reduced myogenic tone (MT) upon increasing pressure by step. This reduced myogenic tone is correlated with a decreased sensitivity and activity of stretch activated channels (SACs) suggesting a role for SRF in maintaining myogenic tone in small resistance arteries. ${ }^{32}$ The second study explored the impact of SRF absence on larger arteries (carotid and aorta) and more specifically its role in aortic stiffness. ${ }^{33}$ Aortic stiffness is physiologically related to ageing and accelerates atheromatosis in the presence of other risk factors. Increasing aortic stiffness with age has been mainly attributed to changes in both the organization and the content of the extracellular matrix (ECM) as well as changes in the structural properties of VSMCs. As expected SRF deficient arteries showed a decrease in the expression of contractile protein such as smooth muscle actin and myosin light chain. Several proteins involved in cell-matrix interaction such as integrin $\alpha \mathrm{V}$ and integrin $\beta 3$ were also downregulated. ${ }^{33}$ These modifications in protein expression were suggested to be responsible for the physiological alterations observed in mice. Indeed, the absence of SRF in VSMCs leads to a reduced arterial stiffness suggesting that SRF upregulation during ageing could participate in the age related arterial stiffness. Taken together these in vivo studies highlight an essential regulatory role of SRF in the function and structure of elastic and resistance arteries and their remodelling during ageing.

\section{SRF and skeletal muscle}

\section{SRF during skeletal muscle formation and postnatal growth}

Skeletal muscle of the limb and trunk derive essentially from mesodermal structures called somites and more specifically form the dorsal part of these structures. Following an initial differentiation step under the influence of myogenic determination factors (Myf5, MyoD, Myogenin) to form the myotome, these cells delaminate and migrate into distant sites such as the limb to fuse and form multinucleated muscle fibres. ${ }^{34,35}$ A potential role for the involvement of SRF in myogenesis was first inferred by in vitro experiments where the authors showed the requirement of this factor for MyoD expression and consequently myogenic differentiation and maintenance of muscle fibres. ${ }^{36}$ However, subsequent in vivo studies using early (Myo-Cre) and late (Mck-Cre) Cre expressing lines to delete SRF in myogenic cells failed to block MyoD expression, myoblast formation and fusion. These mice showed a compromised hypertrophic growth necessary for the formation of fully functional skeletal muscle and led to early postnatal lethality probably due to respiratory failure. These results suggest that the $S r f$ mutant phenotype reflects a late function of SRF in hypertrophic growth rather than an early developmental role. ${ }^{37}$ The use of Cre expressing line during early myogenic commitment such as Pax3Cre would be of use to rule out or confirm the implication of SRF in the early events of myoblast formation or fusion. During the postnatal period, skeletal muscle achieves its growth by extensive synthesis of structural and contractile proteins and the 


\section{Serum Response Factor in muscle tissues}

Eur J Transl Myol 26 (2): 87-92

fusion of myogenic stem cells to the existing multinucleated muscle fibres. Secreted factors such as Igf- 1 and Interleukin 4 contribute to the growth of the muscle. The role of SRF during this growth phase was also investigated in vivo using the human skeletal actin promoter to drive Cre recombinase expression. Mutant mice displayed growth retardation and a major decrease in muscle mass. A sharp decrease in skeletal actin and other contractile genes was also evident in these mice. Moreover, myofibres lacking SRF displayed a reduced myonuclear number and failed to regenerate suggesting a possible defect in satellite cell recruitment probably via the downregulation of transcription IL-4 and IGF-1 genes. $^{38}$

\section{SRF in skeletal muscle homeostasis and ageing}

Under the influence of external or intrinsic cues, adult skeletal muscle can change its size, mass and undergo reparation following injury-induced damage. This plasticity could be compromised in pathological conditions such as tumour-induced muscle cachexia or defects due to genetic mutations in structural genes such as the dystrophin. ${ }^{39,40}$ Ageing also reduces the ability of skeletal muscle for reparation and is characterised by reduced force generation, sarcopenia and fibrosis. ${ }^{41,42}$ Several lines of evidence suggest the implication of SRF in modulating muscle plasticity. Contrary to the cardiac muscle, SRF is downregulated during ageing in human and mice skeletal muscle suggesting that this naturally occurring decrease in could contribute to the muscle phenotype observed during the ageing process and in sarcopenia. ${ }^{43}$ Inducing SRF loss in adult myofibers did not produce an overt phenotype within the first months after triggering $S R F$ disruption suggesting that under normal physiological conditions SRF is not implicated in muscle homeostasis. However, after several months, mutant skeletal muscles develop a wide spectrum of alterations including atrophy, fibrosis, lipid accumulation and a perturbed regeneration. In combination, all these features are characteristic of aged skeletal muscle. ${ }^{43}$ Furthermore, the absence of SRF in muscle fibres led to altered regenerative response and accumulation of fibrosis suggesting a that SRF is required for the maintenance of an adequate "niche" for the efficient recruitment of muscle satellite cells. The defect in satellite cell recruitment in SRF muscle deficient mice was further confirmed in a different experimental setting where mutant mice failed to respond to overload induced hypertrophy and identified $\mathrm{SRF}$ as a central player in a I16, Il4, and Cox 2 gene network. ${ }^{44}$ It could be interesting to further investigate the role of SRF in skeletal muscle biology and more specifically the role of this factor in muscle stem cells using for instance satellite cell specific Cre expressing mice (Pax7-Cre). Its role in a pathological setting such as cancer-induced muscle cachexia model would also be of interest. $^{45}$

\section{Contributions}

DC read and edited the manuscript, ND wrote the manuscript, $\mathrm{MH}$ collected biliographic data for the manuscript, ZL Wrote the manuscript, Generated floxed SRF mice and data related to the cardiac and vascular phenotypes, AP Wrote the manuscript, Generated floxed SRF mice and data related to the cardiac and vascular phenotypes.

\section{Acknowledgement}

This work was supported by AFM grants (no. 20120773; no. 2014-17689) and Emergence UPMC (no. 2011-EME1119).

\section{Conflict of Interest}

The authors declare no conflict of interests.

\section{Corresponding Author}

Ara Parlakian, Sorbonne University - UPMC Department of Biological Adaptation and Ageing, IBPS, UMR 8256 CNRS, INSERM U1164 Genetics and Physiology of Muscle Tissues. 7, quai Saint Bernard case 256, Bat A 6eme étage, 75252 Paris Cedex 5 France. E-mail: ara.parlakian@upmc.fr

\section{E-mails of coAuthors}

DarioColetti: dario.coletti@upmc.fr

Nissrine Daou: daou.nissrine@gmail.com

Medhi Hassani: medhihsn@gmail.com

Zhenlin Li: zhenlin.li@upmc.fr

\section{References}

1. Davis JL, Long X, Georger MA et al. Expression and comparative genomics of two serum response factor genes in zebrafish. Int J Dev Biol 2008; 52:389-96.

2. Posern G, Treisman R. Actin' together: serum response factor, its cofactors and the link to signal transduction. Trends Cell Biol 2006;16:588-96.

3. Huet A, Parlakian A, Arnaud $\mathrm{MC}$ et al. Mechanism of binding of serum response factor to serum response element. FEBS J 2005;272:3105-19.

4. Sun Q, Chen G, Streb JW et al. Defining the mammalian CArGome. Genome Res 2006 ; 16:197-207.

5. Olson EN, Nordheim A. Nat Rev Mol Cell Biol 2010;11:353-65.

6. Miralles F, Posern G, Zaromytidou AI, Treisman R. Actin dynamics control SRF activity by regulation of its coactivator MAL. Cell 2003;113:329-42.

7. Sotiropoulos A, Gineitis D, Copeland J, Treisman R.Signal-regulated activation of serum response factor is mediated by changes in actin dynamics. Cell 1999;98:159-69. 


\section{Serum Response Factor in muscle tissues}

Eur J Transl Myol 26 (2): 87-92

8. Zhang X, Azhar G, Helms SA, Wei JY. Regulation of cardiac microRNAs by serum response factor. $\mathbf{J}$ Biomed Sci 2011;18:15.

9. Park C, Hennig GW, Sanders KM et al. Serum response factor-dependent MicroRNAs regulate gastrointestinal smooth muscle cell phenotypes. Gastroenterology 2011;141:164-75.

10. Arsenian S, Weinhold $\mathrm{B}$, Oelgeschläger $\mathrm{M}$ et al. Serum response factor is essential for mesoderm formation during mouse embryogenesis. EMBO J $1998 ; 17: 6289-99$.

11. Parlakian A, Tuil D, Hamard G, et al. Targeted inactivation of serum response factor in the developing heart results in myocardial defects and embryonic lethality. Mol Cell Biol 2004;24:528189.

12. Wiebel FF, Rennekampff V, Vintersten K, et al. Generation of mice carrying conditional knockout alleles for the transcription factor SRF. Genesis 2002;32:124-26.

13. Miano JM, Ramanan N, Georger MA, et al. Restricted inactivation of serum response factor to the cardiovascular system. Proc Natl Acad Sci USA 2004;101:17132-37.

14. Sedmera D, Pexieder T, Vuillemin $M$ et al. Developmental patterning of the myocardium. Anat. Rec 2000 ;258:319-37.

15. Niu Z, Yu W, Zhang SX, et al. Conditional mutagenesis of the murine serum response factor gene blocks cardiogenesis and the transcription of downstream gene targets. J Biol Chem 2005;280:32531-38.

16. Davis FJ, Gupta M, Pogwizd SM, et al. Increased expression of alternatively spliced dominant negative isoform of SRF in human failing hearts. Am J Physiol Heart Circ Physiol 2002;282:H152133.

17. Chang $\mathrm{J}$, Wei L, Otani $\mathrm{T}$, et al. Inhibitory cardiac transcription factor, SRF-N, is generated by caspase 3 cleavage in human heart failure and attenuated by ventricular unloading. Circulation 2003;108:40713.

18. Parlakian A, Charvet C, Escoubet B, et al. Temporally controlled onset of dilated cardiomyopathy through disruption of the SRF gene in adult heart. Circulation 2005;112:2930-39.

19. Diguet N, Mallat Y, Ladouce R et al. Muscle creatine kinase deficiency triggers both actin depolymerization and desmin disorganization by advanced glycation end products in dilated cardiomyopathy. J Biol Chem 2011;286:35007-19.

20. Takahashi T, Schunkert H, Isoyama S et al. Agerelated differences in the expression of protooncogene and contractile protein genes in response to pressure overload in the rat myocardium. J Clin Invest 1992;89:939-46.
21. Lu XG, Azhar G, Liu L et al. SRF binding to SRE in the rat heart: influence of age. J Gerontol A Biol Sci Med Sci 1998;53:B3-10.

22. Angelini A, Li Z, Mericskay M, Decaux JF. Regulation of Connective Tissue Growth Factor and Cardiac Fibrosis by an SRF/MicroRNA-133a Axis. PLoS One 2015;10.

23. Zhang X, Azhar G, Furr MC, et al. Model of functional cardiac aging: young adult mice with mild overexpression of serum response factor. Am J Physiol Regul Integr Comp Physiol 2003;285:R552-60.

24. Zhang X, Azhar G, Chai J, et al. Cardiomyopathy in transgenic mice with cardiac-specific overexpression of serum response factor. Am $\mathbf{J}$ Physiol Heart Circ Physiol 2001;280:H1782-92.

25. Gary-Bobo G, Parlakian A, Escoubet B, et al. Mosaic inactivation of the serum response factor gene in the myocardium induces focal lesions and heart failure. Eur J Heart Fail 2008;10:635-45.

26. Parmacek MS. Transcriptional programs regulating vascular smooth muscle cell development and differentiation. Curr Top Dev Biol 2001;51:69-89.

27. Gomez D, Swiatlowska P, Owens GK. Epigenetic Control of Smooth Muscle Cell Identity and Lineage Memory. Arterioscler Thromb Vasc Biol $2015 ; 35: 2508-16$

28. Franco CA, Mericskay M, Parlakian A, et al. Serum response factor is required for sprouting angiogenesis and vascular integrity. Dev Cell 2008; 15:448-61.

29. Franco CA, Li Z.SRF in angiogenesis: branching the vascular system. Cell Adh Migr 2009;3:264-7.

30. Mericskay M, Blanc J, Tritsch E, et al. Inducible mouse model of chronic intestinal pseudoobstruction by smooth muscle-specific inactivation of the SRF gene. Gastroenterology 2007; 133:1960-70.

31. Lacolley P1, Regnault V, Nicoletti A et al. The vascular smooth muscle cell in arterial pathology: a cell that can take on multiple roles. Cardiovasc Res 2012 ;95:194-204.

32. Retailleau K, Toutain B, Galmiche $\mathrm{G}$ et al. Selective involvement of serum response factor in pressure-induced myogenic tone in resistance arteries. Arterioscler Thromb Vasc Biol 2013 ;33:339-46.

33. Galmiche $\mathrm{G}$, Labat $\mathrm{C}$, Mericskay $\mathrm{M}$ et al. Inactivation of serum response factor contributes to decrease vascular muscular tone and arterial stiffness in mice. Circ Res 2013;112:1035-45.

34. Buckingham M, Rigby PW. Gene regulatory networks and transcriptional mechanisms that control myogenesis. Dev Cell 2014;28:225-38.

35. Buckingham M, Relaix F.PAX3 and PAX7 as upstream regulators of myogenesis. Semin Cell Dev Biol 2015;44:115-25. 


\section{Serum Response Factor in muscle tissues}

Eur J Transl Myol 26 (2): 87-92

36. Gauthier-Rouviere C, Vandromme M, Tuil D et al. Expression and activity of serum response factor is required for expression of the muscle-determining factor MyoD in both dividing and differentiating mouse C2C12 myoblasts. Mol Biol Cell 1996;7:719-29.

37. Li S, Czubryt MP, McAnally J, et al. Requirement for serum response factor for skeletal muscle growth and maturation revealed by tissue-specific gene deletion in mice. Proc Natl Acad Sci USA 2005;102:1082-87.

38. Chavret C, Houbron C, Parlakian A, et al. New role for serum response factor in postnatal skeletal muscle growth and regeneration via the interleukin 4 and insulin-like growth factor 1 pathways. Mol Cell Biol 2006;26:6664-74.

39. Egerman MA, Glass DJ. Signaling pathways controlling skeletal muscle mass. Crit Rev Biochem Mol Biol 2014;49:59-6
40. Fearon KC, Glass DJ, Guttridge DC. Cancer cachexia: mediators, signaling, and metabolic pathways. Cell Metab 2012;16:153-66.

41. Sousa-Victor P, Muñoz-Cánoves P. Regenerative decline of stem cells in sarcopenia. Mol Aspects Med 2016;xxx:yy_zz.

42. Brack AS, Muñoz-Cánoves P. The ins and outs of muscle stem cell aging. Skelet Muscle 2016;18:61

43. Lahoute C, Sotiropoulos A, Favier M, et al. Premature aging in skeletal muscle lacking serum response factor. PLoS One 2008;3:e3910.

44. Guerci A, Lahoute C, Hébrard S et al. Srfdependent paracrine signals produced by myofibers control satellite cell-mediated skeletal muscle hypertrophy. Cell Metab 2012;15:25-37.

45. Aulino P, Berardi E, Cardillo VM et al. Molecular, cellular and physiological characterization of the cancer cachexia-inducing C26 colon carcinoma in mouse. BMC Cancer 2010;10:363. 